\title{
9 Heisenberg's Uncertainty Principle (aka Indeterminacy)
}

There exists in nature pairs of properties of things, also called parameters or attributes, such as values of speed or time durations or location, which cannot be known with arbitrarily high precision when they pertain to a same object. Such quantities are incompatible at the limit - meaning that the level of accuracy with which we can know and measure one of the attributes is limited and directly affected by how precisely we know the other attribute within the pair. For instance, we cannot know with an arbitrarily high level of accuracy an object's speed and its geographical location at the same time. If we need to increase the level of precision with which we measure, say, the object's instantaneous speed, this will come at the cost of decreased accuracy in our knowledge of its instantaneous location.

This 'fuzziness' hasnothing to do withaninabilitytocarryout propermeasurements: it is built-in within nature. With an ideally perfect measuring apparatus, we still could not reach an arbitrarily high accuracy for both measurements. Properties that belong within a pair of attributes that are 'incompatible at the limit' are sometimes called 'orthogonal', or alternatively, 'conjugate' attributes.

Here's an example: let's take two attributes of a sheet of paper - its colour and its surface area. These two properties are orthogonal. Indeed, take the sheet of paper and cut it in half. Then again. And again, and again, and so forth... There will fast come a time when what is left of the sheet has become too small to be able to tell its colour: all that's left to see is a tiny, darkish little dot of paper. This is in no way because of imperfect sight which could be improved by, say, the use of a microscope; if we keep cutting the shrinking paper remnant again and again there will eventually come a time when its dimensions are smaller than the amplitude of the light rays needed to reflect and convey the paper's colour. There is a threshold of compatibility, a certain value of the surface area, below which we simply cannot tell the colour - or in other words, there exists an incompatibility at the limit between size and colour, below a certain value threshold. The thresholds' numerical values depend on the particular attributes. For instance, in the above example the precise surface area from which the colour would become indistinguishable depends in some measure upon the particular relative colour.

Heisenberg's Uncertainty Principle expresses the theoretical thresholds and establishes the theoretical limits to our ability to know the value of one attribute depending on the precision with which we know the other (39).

There are many such pairs of incompatible attributes in nature. John Barrow gives an example of the odd consequences which the orthogonality of speed and geographical location can bring about in a billiards game. Should one play exactly the same game - hitting the balls at exactly the same spot with exactly the same energy imparting the exact same speed, with exactly the same cue and everything else being exactly the same - the billiards game will still become a totally different game after the very first cue stroke. After a mere twelve cue strokes, the theoretical cumulative uncertainty attached to the location of the ball becomes bigger than the width of the billiards table itself. 
The French astronomer and mathematician Pierre Simon Laplace (born 1827), famously once said that if we were to know all of the universe's atoms and constituents' exact locations and exact momentums at any particular point in time, we would then in principle be able to compute and predict with infinite accuracy the state of the universe at any arbitrary point in time henceforth - if we only had the requisite computing power at our disposal. We now know this to be just plain wrong: with an infinitely precise snapshot of the state of the universe at any point in time, we could not in principle determine the exact state of the universe even one split second later, let alone much later - it's fundamentally and intrinsically impossible to do so (40).

An interesting feature of this real-world uncertainty is that it allows all kinds of phenomena as long as these phenomena remain 'under the radar' of direct perception by reality itself, as it were. For instance, because span of time and mass are orthogonal attributes, unseen 'virtual' particles and masses continuously pop in and out of existence, and can keep existing as long as they remain in their virtual existence for a shorter time than the associated orthogonal duration of time. The existence of these virtual particles is well established. They give rise to all kinds of real-world phenomena through their indirect effects (41). This all-pervading 'sea', or froth, of virtual particles and masses popping in and out of existence is often called the 'quantum foam'. As we shall see, the existence of this quantum foam entails all kinds of measurable effects and real life phenomena even in our everyday macroscopic reality. In fact, as we shall see, it goes much further than that; macroscopic reality itself is an emergent phenomenon from quantum foam, sometimes therefore called pre-reality. We'll revisit this issue later. 